\title{
Urban Traffic Control Problem: a Game Theory Approach 1
}

\author{
Israel Alvarez Villalobos ${ }^{2}$ Alexander S. Poznyak \\ Alejandro Malo Tamayo \\ CINVESTAV-IPN, Departamento de Control Automático, A.P. \\ 14-740, CP 07000, México, D.F. \\ e-mail: ialvarez@ctrl.cinvestav.mx
}

\begin{abstract}
Traffic congestion is an issue in every major city. Many approaches have been tried. In this paper, we propose to model signalized intersections as finite controlled Markov chains. The intersection represents a noncooperative game where each player try to minimize its queue, so $\epsilon-$ Nash's equilibrium is the solution. This paper is focused on the traffic light control problem for urban traffic, using Game Theory and Extraproximal Method for its realization. The examples show the effectiveness of the suggested approach.
\end{abstract}

Keywords: Traffic lights control; Game Theory; Markov Chains; Simulation.

\section{INTRODUCTION}

Among many approaches dealing with the urban intersections problem, some of them consider isolated intersections and other consider groups of intersections. The former case is considered in Allsop [1971, 1976], Impotra [2002], Impotra and Cantarella [1984] and Sen and Head [1997] the latter case is talked in Little et al. [1981], Little [1966] Robertson [1969], Boillot et al. [1992] and McDonald and Hounsell [1991].

Allsop [1971], Allsop [1976], Impotra and Cantarella [1984], Impotra [2002] aim to determine the phases that minimize the total delay or maximize the intersection capacity. Sen and Head [1997] optimizes the time splits at an intersection using a global minimization of binary variables on a large horizon.

The synchronization of the lights on several intersections of a street is done using MAXBAND proposed in Little et al. [1981], Little [1966] or TRANSYT developed in Robertson [1969], Boillot et al. [1992] and McDonald and Hounsell [1991]. The adaptive version of TRANSYT: SCOOT, see Hunt et al. [1982], Robertson and Bretherton [1991], McDonald and Hounsell [1991] and Boillot et al. [1992] is used worldwide. A more detailed recent review of control traffic techniques can be found in Papageorgiou et al. [2003]. The game theory was used by Chen O. J. [1998] and Bel [2000].

The traffic ligths in Mexico city are controlled using a cooperative scheme. However, noncooperation drivers, and a noncooperative behavior between drivers and signals is frequently observed. Mexico City's net is constituted by $\mathbf{3 0 7 6}$ intersections with traffic lights of which there are $\mathbf{3 1 4}$ conflicting cruises. The main cause of the saturation is the imbalance between the demand and the supply of services in the metropolitan area. A detailed

1 Work partially supported by the SEP-CONACYT 47048 Project.

2 Work supported by a CONACYT Ph.D. grant description of Mexico City is presented in del Gobierno del Distrito Federal [2002].

A landmark in noncooperative games is the equilibrium introduced in Nash [1950]. Rosen in a seminal paper Rosen [1965], introduces the numerical methods to find the noncooperative constrained Nash equilibrium. The proposed construction does not require the "Strictly Diagonal Convexity Condition" as in Rosen [1965], and use the work of Godoy Godoy et al. [2003] that deals with a multi-participant dynamic (averaged) non cooperative finite game with constraints where each player has a finite number of actions and a finite number of states; the complete information on the corresponding payoff and constraints is assumed to be available. This paper consider the complete information case and follows the lead of Antipin [2005a], Antipin [2005b]. Here the extraproximal method is applied with an additional regularizing term that permits its convergence to one of Nash equilibrium points. Each player has a finite number of actions and a finite number of states. The complete information on the corresponding payoff and transition matrices is assumed to be available.

This work is structured as follows, first we present concepts associated with the proposed model, next we define the game problem, and propose a method to obtain a solution or $\epsilon$-Nash equilibrium point. After this, the result for an intersection with several players is presented with a comparison to a adaptive control.

\section{MARKOV CHAIN MODEL}

The simplest intersection is formed by the crossing of two one-way streets. The vehicle flow is controlled by a two color light $u \in 1 \geq \mathbb{R}^{2} \geq 0$, where $u(1)$ represents the red light and $u(2)$ is the green light. At time $t$, see figure (1), the total number of cars in the street is $x_{t}$, the number of cars entering it is $\xi_{t}$, and the number of cars exiting is $\nu_{t}$; the street has a maximum capacity of $n \mid x^{+}$. The dynamics of the vehicle flow at one street is described by 
the following equations

$$
\begin{gathered}
u_{t}=u(1): \text { red light } \\
x_{t+1}= \begin{cases}x^{+} & : \xi_{t}+x_{t}>x^{+} \\
\xi_{t}+x_{t} & : \xi_{t}+x_{t} \leq x^{+}\end{cases} \\
u_{t}=u(2): \text { green light } \\
x_{t+1}= \begin{cases}x^{+} & : \xi_{t}+x_{t}-\nu_{t}>x^{+} \\
\xi_{t}+x_{t}-\nu_{t} & : 0 \leq \xi_{t}+x_{t}-\nu_{t} \leq x^{+}\end{cases}
\end{gathered}
$$

so a street can be seen as a finite capacity FIFO buffer or queue. We assume that the input flow is a Poisson

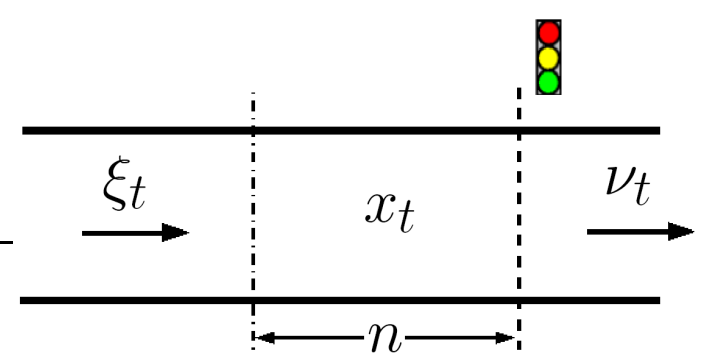

Fig. 1. Flow variables.

Process with parameter $\lambda_{\xi}$. Each control strategy defines a transition matrix $\Pi_{j i}^{u_{t}}$ for the controlled Markov chain:

For the red light:

$$
\begin{gathered}
\Pi_{j i}^{u(1)}=P\left\{x_{t+1}=i \mid x_{t}=j \wedge u_{t}=u(1)\right\} \\
\Pi_{j i}^{u(1)}=\delta_{i, x^{+}}\left(1-\sum_{s=0}^{x^{+}-j} \frac{e^{-\lambda} \lambda^{s}}{s !}\right)+ \\
+\sum_{s=0}^{i-j} \frac{e^{-\lambda} \lambda^{s}}{s !} \chi(s \geq 0)\left(\sum_{s=0}^{x^{+}-j} \frac{e^{-\lambda} \lambda^{s}}{s !}\right)
\end{gathered}
$$

For green light:

$$
\begin{gathered}
\Pi_{j i}^{u(2)}=P\left\{x_{t+1}=i \mid x_{t}=j \wedge u_{t}=u(2)\right\} \\
\Pi_{j i}^{u(2)}=\delta_{i, x^{+}}\left(1-\sum_{k=0}^{x^{+}} \frac{e^{-\lambda} \lambda^{\left(x^{+}-j+k\right)}}{\left(x^{+}-j+k\right) !} P_{\nu_{t}=k}\right)+ \\
+\sum_{k=0}^{x^{+}}\left[\frac{e^{-\lambda} \lambda^{(i-j+k)}}{(i-j+k) !} P_{\nu_{t}=k}\left(\sum_{s=k-j}^{x^{+}+k-j} \frac{e^{-\lambda} \lambda^{s}}{s !} \chi(s \geq 0)\right)\right]
\end{gathered}
$$

Associating with the two control strategies $P\left(u_{i}^{1}\right)=$ $d_{i}^{1}, P\left(u_{i}^{2}\right)=d_{i}^{2}$ we have:

$$
\begin{gathered}
\Pi_{i j}(d):=\pi_{i j}^{(1)} \cdot d_{i}^{1}+\pi_{i j}^{(2)} \cdot d_{i}^{2}=\sum_{k=1}^{2} \pi_{i j}^{k} d_{i}^{k} \\
\Pi_{i j}(d):=\pi_{i j}^{(1)} \cdot d_{i}^{1}+\pi_{j i}^{(2)} \cdot\left(1-d_{i}^{1}\right)
\end{gathered}
$$

with:

$$
\begin{aligned}
& d_{i}^{1} \geq 0, \quad d_{i}^{2} \geq 0 \quad d_{i}^{1}+d_{i}^{2}=1 \\
& d=\left\|\bar{d}_{i}^{k}\right\|_{\substack{i=0, \ldots \\
k=1,2}} \quad d_{i}=\left(d_{i}^{1}, d_{i}^{2}\right) \in S^{2}
\end{aligned}
$$

where $\pi_{i_{l}, j_{l}}^{k_{l}}$ is the transition probability for player $l$ to go from state $j$ to state $i$ with the control action $k$ and $d_{i}^{1}$ is the red light strategy at $i-t h$ state.

\section{THE GAME DESCRIPTION}

The simplest game considers a two one-way-street intersection. So the conflict appears when each player wants to minimize its queue, see figure (2). The extended probability vector will be used in the payoff function with the constraints on the behavior in the model. The use of counter-coalition variables is considered here in order to obtain the equivalent LPP (Linear Programming Problem) and its solution, i.e., the Nash equilibrium point, see Nash [1950], Rosen [1965]. For this intersection we have the following matrices:

First player

Second player

$$
\begin{aligned}
& \Pi_{i_{1}, j_{1}}^{1_{1}}=\Pi_{j i}^{u(1)} \\
& \Pi_{i_{1}, j_{1}}^{2_{1}}=\Pi_{j i}^{u(2)}
\end{aligned}
$$

$$
\begin{aligned}
& \Pi_{i_{2}, j_{2}}^{1_{2}}=\Pi_{j i}^{u(1)} \\
& \Pi_{i_{2}, j_{2}}^{2_{2}}=\Pi_{j i}^{u(2)}
\end{aligned}
$$

Following Gómez et al. [2003], the individual aim of each player can be formulated as follows:

$$
\begin{aligned}
& V_{1}(d)=\sum_{i_{1}=1}^{n} i_{1} P_{i_{1}}\left(d_{i_{1}}, d_{i_{2}}\right) \rightarrow \min _{d_{i_{1}}^{k_{1}} \in S^{2}} \\
& V_{2}(d)=\sum_{i_{2}=1}^{n} i_{2} P_{i_{2}}\left(d_{i_{1}}, d_{i_{2}}\right) \rightarrow \min _{d_{i_{2}}^{k_{2}} \in S^{2}}
\end{aligned}
$$

where for stationary strategies $d_{i_{k}}^{k_{k}}(n)=d_{i_{k}}^{k_{k}}$ and ergodic Markov chains, see Poznyak and Najim [2000] the following identities hold

$$
\begin{aligned}
& P_{i_{1}}\left(d_{i_{1}}, d_{i_{2}}\right)=\sum_{i_{1}=1}^{n} \sum_{i_{2}=1}^{n} \sum_{k_{1}=1}^{2} \sum_{k_{2}=1}^{2} \pi_{i_{1} j_{1}}^{k_{1}} d_{i_{1}}^{k_{1}} p_{i_{1}} d_{i_{2}}^{k_{2}} p_{i_{2}} \\
& P_{i_{2}}\left(d_{i_{1}}, d_{i_{2}}\right)=\sum_{i_{1}=1}^{n} \sum_{i_{2}=1}^{n} \sum_{k_{1}=1}^{2} \sum_{k_{2}=1}^{2} \pi_{i_{2} j_{2}}^{k_{2}} d_{i_{1}}^{k_{2}} p_{i_{1}} d_{i_{2}}^{k_{2}} p_{i_{2}}
\end{aligned}
$$

\subsection{Model in C-variables}

The above problem can be reformulated as linear programming problem by replacing the variables used. Set the variables $c_{i_{1}}^{k_{1}}$ and $c_{i_{2}}^{k_{2}}$ as follow:

$$
c_{i_{1}}^{k_{1}}=d_{i_{1}}^{k_{1}} p_{i_{1}} \quad c_{i_{2}}^{k_{2}}=d_{i_{2}}^{k_{2}} p_{i_{2}}
$$

The allowed strategies will be limited by the constraints

- each vector $c^{k}$ means a stationary mix-strategy and belongs to simplex $S^{(n k)}$ defined by

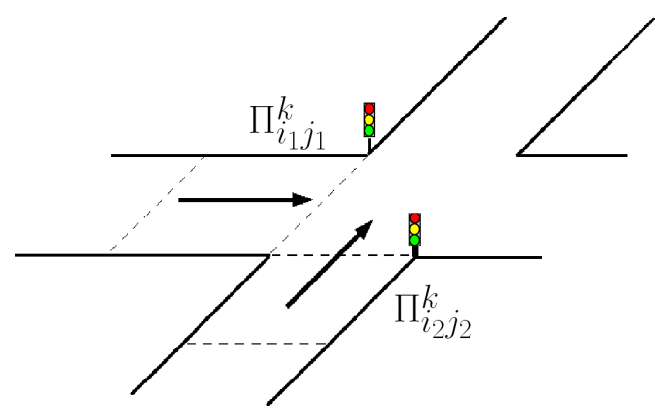

Fig. 2. Flow dynamics of the intersection. 


$$
S^{(n k)}:=\left\{\begin{array}{c}
c^{k} \in R^{n \cdot k} \mid c_{i_{1}}^{k_{1}} \geq 0, c_{i_{2}}^{k_{2}} \geq 0 \\
\sum_{i_{1}=1}^{n} \sum_{k_{1}=1}^{2} c_{i_{1}}^{k_{1}}=1, \sum_{i_{2}=1}^{n} \sum_{k_{2}=1}^{2} c_{i_{2}}^{k_{2}}=1
\end{array}\right\}
$$

- The joint strategy variable $c$ satisfies the ergodicity constraints and belongs to the convex, closed and bounded set $Q_{\text {erg }} \subset R^{2}$ defined as

$$
\begin{gathered}
Q_{e r g}:=Q_{e r g}^{1} \times Q_{e r g}^{2} \\
Q_{e r g}^{n}:=Q_{e r g}^{1,1} \times Q_{\text {erg }}^{1,2} \times \cdots \times Q_{\text {erg }}^{n, 1} \times Q_{\text {erg }}^{n, 2} \\
Q_{\text {erg }}^{n, k}:=\left\{\begin{array}{c}
c_{i_{1}}:=\left(c_{1}, \ldots, c_{n}\right)^{T} \in R^{2} \mid \\
\sum_{k_{1}=1}^{2} c_{i_{1}}^{k_{1}}=\sum_{i_{1}=1}^{n} \sum_{k_{1}=1}^{2} \pi_{i_{1}, j_{1}}^{k_{1}} c_{i_{1}}^{k_{1}} \\
c_{i_{2}}:=\left(c_{1}, \ldots, c_{n}\right)^{T} \in R^{2} \mid \\
\sum_{k_{2}=1}^{2} c_{i_{2}}^{k_{2}}=\sum_{i_{2}=1}^{n} \sum_{k_{2}=1}^{2} \pi_{i_{2}, j_{2}}^{k_{2}} c_{i_{2}}^{k_{2}}
\end{array}\right\}
\end{gathered}
$$

For ergodic Markov chains (see Poznyak and Najim [2000]) one has

$$
\begin{array}{ll}
p_{i_{1}}=\sum_{k_{1}=1}^{2} c_{i_{1}}^{k_{1}}>0 & d_{i_{1}}^{k_{1}}=c_{i_{1}}^{k_{1}} / \sum_{k_{1}=1}^{2} c_{i_{1}}^{k_{1}} \\
p_{i_{2}}=\sum_{k_{2}=1}^{2} c_{i_{2}}^{k_{2}}>0 & d_{i_{2}}^{k_{2}}=c_{i_{2}}^{k_{2}} / \sum_{k_{2}=1}^{2} c_{i_{2}}^{k_{2}}
\end{array}
$$

\subsection{Nash Equilibrium}

The regularized payoff function $V_{(k)}(c)$ for each player depends on players strategies that is,

$$
\begin{aligned}
V_{\delta}^{(1)}(c) & =\sum_{i_{1}=1}^{n} \sum_{i_{2}=1}^{n} \sum_{k_{1}=1}^{2} \sum_{k_{2}=1}^{2} i_{1} c_{i_{1}}^{k_{1}} c_{i_{2}}^{k_{2}}+\frac{\delta}{2}\left\|c^{k_{1}}\right\|^{2} \\
V_{\delta}^{(2)}(c) & =\sum_{i_{1}=1}^{n} \sum_{i_{2}=1}^{n} \sum_{k_{1}=1}^{2} \sum_{k_{2}=1}^{2} i_{2} c_{i_{1}}^{k_{1}} c_{i_{2}}^{k_{2}}+\frac{\delta}{2}\left\|c^{k_{2}}\right\|^{2}
\end{aligned}
$$

Using the stationary mixed strategies

$$
d_{i_{1}}^{k_{1}}=c_{i_{1}}^{k_{1}} / \sum_{k_{1}=1}^{2} c_{i_{1}}^{k_{1}}, d_{i_{2}}^{k_{2}}=c_{i_{2}}^{k_{2}} / \sum_{k_{2}=1}^{2} c_{i_{2}}^{k_{2}}
$$

each player wants to minimize his penalties (in this case, the number of waiting cars) within the associated constraints. Sure, both aims are in conflict which can be resolved by the Nash-equilibrium concept.

The Nash equilibrium point $c^{*}$ in the case of two players is given by the matrix $c$ that satisfies:

$$
c^{k, *} \in \operatorname{Arg} \min _{c^{k} \in S^{(n k)}}\left\{\begin{array}{l}
V_{k}\left(c^{l, *}, c^{k}\right)+\frac{\delta}{2}\left\|c^{k}\right\|^{2} \\
\mid\left(c^{l, *}, c^{k}\right) \in Q_{e r g}^{k} \times Q^{k}
\end{array}\right\}
$$

The uniqueness of the Nash equilibrium point is guaranteed by the strict concavity condition (see Rosen [1965], Poznyak and Najim [2002]).

\subsection{Joint Loss Function}

Following the approach in Rosen [1965] and Tanaka and Yokoyama [1991], let us introduce the $\delta$-regularized joint
Loss function $\rho_{\delta}\left(c^{*}, c\right)$ defined by

$$
\begin{gathered}
\rho_{\delta}\left(c, c^{*}\right):=\sum_{k=1}^{N} V_{\delta}^{k}\left(c, c^{*}\right) \\
c^{\top}:=\left(c^{l}, c^{k}\right) \in S \times Q_{\text {erg }} \\
c^{*, \top}:=\left(c^{l, *}, c^{k, *}\right) \in S \times Q_{\text {erg }}
\end{gathered}
$$

for any $c \in S \times Q_{\text {erg }}$.

The fixed point $c^{*} \in S \times Q_{\text {erg }}$ is a Nash equilibrium (see Nash [1950]) in a $N$-person Markov chain game (11) if only if satisfies the equation

$$
c^{*} \in \operatorname{Arg} \min _{c \in S \times Q_{e r g}} \rho_{\delta}\left(c, c^{*}\right)
$$

An equilibrium point $c^{*}$ in (13) can be characterized by the inequality

$$
\rho_{\delta}\left(c, c^{*}\right) \geq \rho_{\delta}\left(c^{*}, c^{*}\right), \delta>0
$$

Theorem 1. (Tanaka and Yokoyama [1991]). A strategy $c^{*}$ $\in S \times Q_{\text {erg }}$ is a Nash equilibrium point (in the sense 11) in N-person finite Markov chain game (10) if and only if

$$
\min _{c \in S \times Q_{\text {erg }}} \rho_{\delta}\left(c, c^{*}\right)=\rho_{\delta}\left(c^{*}, c^{*}\right), \delta>0
$$

\subsection{Extraproximal Procedure for Solving Equilibrium Point}

An "extraproximal method", applied to resolving convex static two-person games, is designed and analyzed in Antipin [2005a] and Antipin [2005b]. Here we will apply it to find a Nash equilibrium $c^{*} \in S \times Q_{\text {erg }}$, defined by (13), in Markov chain finite games (8)-(10). The idea of this method, as it may be applied to this problem, consists of the following "iterative rules" implementation:

(1) The first half-step (prediction):

$$
\begin{gathered}
\bar{c}_{n}^{k}=\arg \min \left\{\frac{1}{2}\left\|c^{k}-c_{n}^{k}\right\|^{2}+\alpha V_{\delta}^{(k)}\left(c^{k}, c_{n}^{\hat{k}}\right)\right\} \\
c^{k} \in S \times Q_{\text {erg }} ; k=1,2 ; n=1, \ldots
\end{gathered}
$$

(2) The second (basic) half-step:

$$
\begin{gathered}
c_{n+1}^{k}=\arg \min \left\{\frac{1}{2}\left\|c^{k}-c_{n}^{k}\right\|^{2}+\alpha V_{\delta}^{(k)}\left(c^{k}, \bar{c}_{n}^{\hat{k}}\right)\right\} \\
c^{k} \in S \times Q_{\text {erg }} ; k=1,2 ; n=1, \ldots
\end{gathered}
$$

where $V_{\delta}^{(k)}\left(c^{k}, \bar{c}_{n}^{\hat{k}}\right)=V_{\delta}^{k}\left(\bar{c}^{1, k}, c^{k}\right)$ and $V_{\delta}^{k}\left(c^{k}, c_{n}^{\hat{k}}\right)=$ $V^{k}\left(c_{n}^{1}, c_{n}^{k}\right)$, with the step size $\alpha$ from a certain fixed interval $0<\alpha<\alpha_{0}$ and a small enough $\delta>0$.

Theorem 2. (Antipin [2005a]). Assume that there exists a solution to problem (13). Then, the sequence $c_{n}$ generated by the extraproximal method (16)-(17) with the step size $\alpha$, chosen from the condition $0<\alpha<\left(\sqrt{2} C_{0}\right)^{-1}$ $\left(C_{0}=\max \left\{C_{k}\right\}, k=\overline{1, N}\right)$, converges monotonically in the euclidean norm to a game equilibrium (one of possible solutions), i.e.,

$$
c_{n}^{k} \rightarrow c^{k, *} \text { as } n \rightarrow \infty
$$

\section{EXPERIMENT RESULTS}

We consider the same average flows for both players. Incoming and outgoing flows $\left(\lambda_{\xi_{t}}^{1}=\lambda_{\xi_{t}}^{2}, \lambda_{\nu_{t}}^{1}=\lambda_{\nu_{t}}^{1}\right)$. This mean that the transition matrices are identical for both 
players. Since the buffer capacity is $x^{+}=n$, we have two $n$ square matrices. Although we can guess the result, solving the linear programming problem for the payoff function (10), the cycle time split that results is

$$
\begin{array}{lll}
d_{i_{1}}^{1}=0.5 & \text { and } & d_{i_{1}}^{2}=0.5 \\
d_{i_{2}}^{1}=0.5 & \text { and } & d_{i_{2}}^{2}=0.5
\end{array}
$$

This is the Nash equilibrium point for two identical players, i.e., with equal transition matrices. It assigns the same time to each participant. This result serves as reference for intersections with unequal players, i.e., with different input/output flows.

For the case when $x^{+}=8, \lambda_{\xi_{t}}=7, \lambda_{\nu_{t}}=4$ for one player and $\lambda_{\xi_{t}}=6, \lambda_{\nu_{t}}=7$ for the other one, the solution of the LPP is:

$$
\begin{array}{lll}
d_{i_{1}}^{1}=0.1571 & \text { and } & d_{i_{1}}^{2}=0.8429 \\
d_{i_{2}}^{1}=0.8429 & \text { and } & d_{i_{2}}^{2}=0.1571
\end{array}
$$

This result could be expected since the first section has a greater load (having a greater input stream and a smaller output flow) with respect to the second section that has a smaller load of automobiles.

\section{MORE GENERAL INTERSECTIONS}

Other kind of intersections can be treated the same way. We now can consider a blade -a three street intersection-, see figure (3).

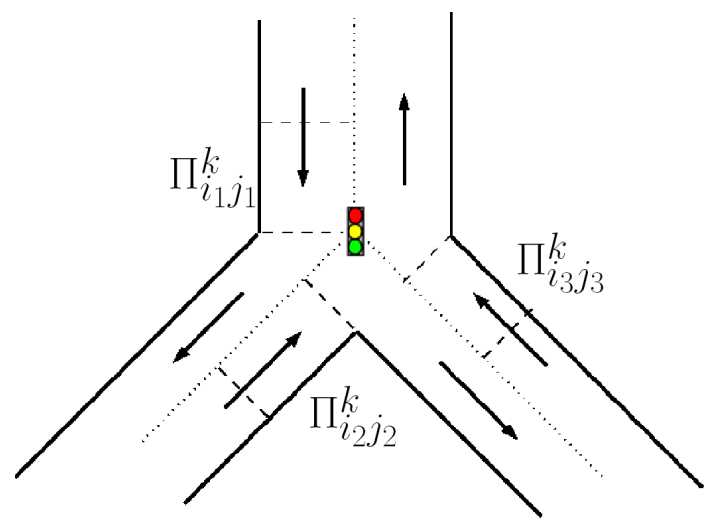

Fig. 3. Flow dynamics of the three player intersection.

The result for identical players in a blade intersection is:

$$
\begin{array}{lll}
d_{i_{1}}^{1}=0.6666 & \text { and } & d_{i_{1}}^{2}=0.3333 \\
d_{i_{2}}^{1}=0.6666 & \text { and } & d_{i_{2}}^{2}=0.3333 \\
d_{i_{3}}^{1}=0.6666 & \text { and } & d_{i_{3}}^{2}=0.3333
\end{array}
$$

Notice that we don't give data of the flows averages (input and output), because the result will be always the same. For different players is necessary to set the data: let $x^{+}=7, \lambda_{\xi_{t}}^{1}=4, \lambda_{\nu_{t}}^{1}=5 ; \lambda_{\xi_{t}}^{2}=7, \lambda_{\nu_{t}}^{2}=5 ; \lambda_{\xi_{t}}^{3}=6$, $\lambda_{\nu_{t}}^{3}=6$. The Nash equilibrium for each player of this game is found to be

$$
\begin{array}{lll}
d_{i_{1}}^{1}=0.9077 & \text { and } & d_{i_{1}}^{2}=0.0923 \\
d_{i_{2}}^{1}=0.2140 & \text { and } & d_{i_{2}}^{2}=0.7860 \\
d_{i_{3}}^{1}=0.8783 & \text { and } & d_{i_{3}}^{2}=0.1217
\end{array}
$$

This result could also be expected since the first player is under saturated $\left(\lambda_{\xi_{t}}^{1}<\lambda_{\nu_{t}}^{1}\right)$ therefore its strategy for green light $d_{i_{1}}^{2}$ is the smallest, the second second player strategy $d_{i_{2}}^{2}$ is greater than third player strategy $d_{i_{3}}^{2}$ because this player (second) is oversaturated $\left(\lambda_{\xi_{t}}^{2}>\lambda_{\nu_{t}}^{2}\right)$, and finally the third player strategy is greater than first player strategy due to its flows are the same $\left(\lambda_{\xi_{t}}^{3}=\lambda_{\nu_{t}}^{3}\right)$.

Until now our streets have only one direction; so in order to get a more realist model we consider opposing flows as participant pairs; it means that we consider the players on the same street as only one player, see figure (4). The following constraints are required in order to avoid

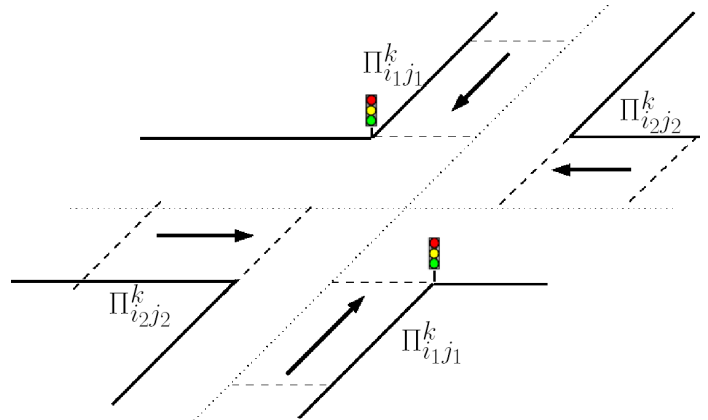

Fig. 4. Flow dynamics of the four flow intersection (two participants)

collisions

$$
\begin{array}{cl}
d_{i_{1}}^{1}=d_{i_{2}}^{2} & d_{i_{2}}^{1}=d_{i_{1}}^{2} \\
d_{i_{1}}^{1}+d_{i_{1}}^{2}=1 & d_{i_{2}}^{1}+d_{i_{2}}^{2}=1
\end{array}
$$

The English Turn its a special case and the control of the traffic lights in this type of crossing looks glance as a complex task in fact for these is not the case. For these turn the passage of vehicles are based on the location of these: in a turn they advance from north to the south and vice versa, in the following turn they advance from east to west and vice versa. The structure of this type of intersection appears in figure (5). The arrows mark

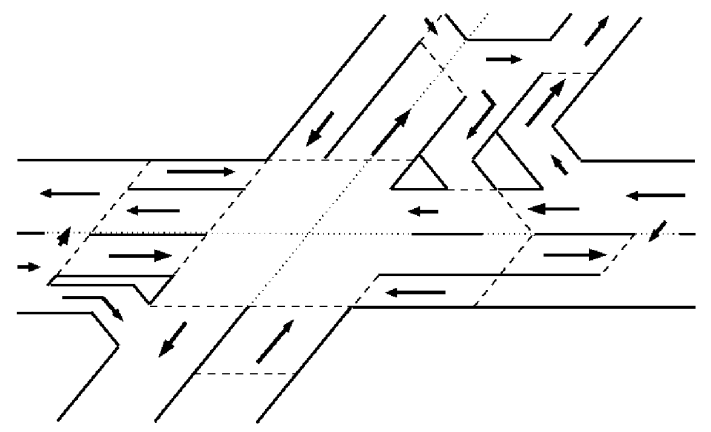

Fig. 5. Flow dynamics of the English turn.

the position of the vehicles within each segment of cars in the intersection, and the players behavior is based on the previously described fact. We considered the segments that go of north to the south and the south to north for the construction of the transition matrices of our first player and the segments that go from east to west and the west to east for the second participant. 


\section{COMPARISON}

We compare our method against the following timevarying adaptive strategy, since there is not way to compare the proposed solution versus a similar algorithm, the following strategy is useful, because characterizes the behavior of the traffic lights in iterative form.

$$
\begin{gathered}
d_{i_{1}}^{k_{k}}=\frac{2}{\left(x_{i_{1}}+1\right) x_{i_{1}}} i, \quad d_{i_{2}}^{k_{k}}=\frac{2}{\left(x_{i_{2}}+1\right) x_{i_{2}}} i \\
i=0,1,2 \ldots, n
\end{gathered}
$$

We consider sixty iterations or cycles, this mean that our strategy will be computed every cycle time (cycle time $=$ green light time + red light time), a buffer size of twentysix cars $x^{+}=26$ for each player (the maximum capacity of the two segments of the street); and random input/output flows $\left(\lambda_{\xi_{t}}^{l}, \lambda_{\nu_{t}}^{l}\right)$ were generated by Poisson distribution with $\lambda_{\xi_{t}}^{1}=20, \lambda_{\nu_{t}}^{1}=21$ for first player and $\lambda_{\xi_{t}}^{2}=19$, $\lambda_{\nu_{t}}^{2}=20$ for the second; all considerations are based on participant pairs.

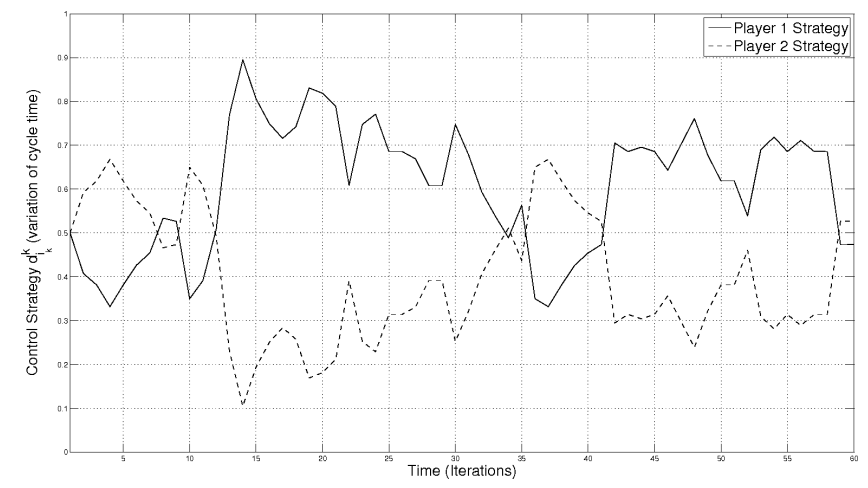

Fig. 6. Game Theory Control.

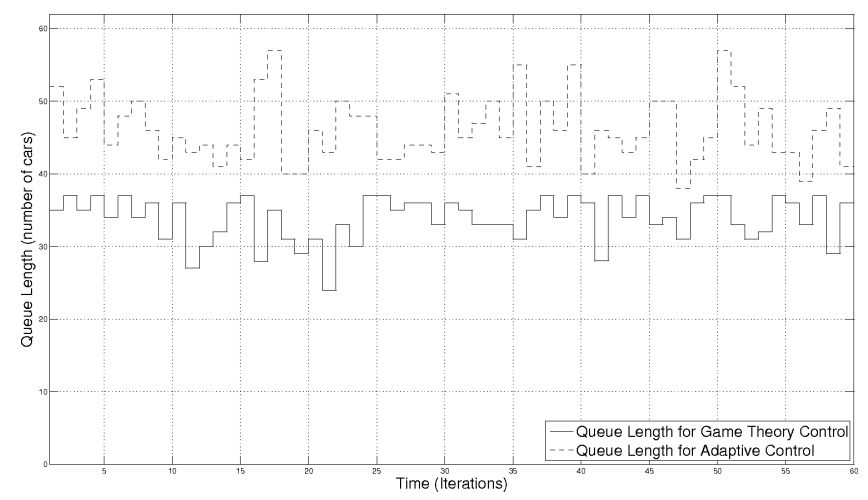

Fig. 7. Queue evolution for the Intersection

Figure (6) shows the green light strategy value for each player, which is the red light strategy for the other player and the figure (7) compares the total queue average for the intersection; between game theory control and adaptive control, we can obtain an improvement of $\mathbf{2 6 . 4 5 \%}$ in performance when using game theory strategy. The result is variable and depends on flow averages; several experiments varing the (input and output) flows gave better results for saturated intersections and gave the same performance for unsaturated intersections.

\section{CONCLUSION}

This paper presents an approach to the urban traffic problem based on game theory and a Markov chain model. This technique is compared with an adaptive control, the results obtained are applied to control the lights cycle time.

At each iteration a game is solved by extraproximal method to find a $\epsilon-\mathrm{Nash}$ equilibrium point. The ergodicity condition is fulfilled; this means that we do not require past information to manage the actual queue length. An improvement over the adaptive control is not always obtained, nevertheless in the worst case, our control technique will work as the adaptive control. This occurs when we have low traffic because the throughput is higher.

In this work, the approach was applied to simple isolated intersections, but was extended to more complex cases like "English" turns. The comparison will be improved using other methods of traffic lights control. The behavior of other types of networks, like computer or communication networks, could be analyzed using this technique. Future work includes to develop a general framework to deal with the intersections on a street or on a streets network.

\section{REFERENCES}

R. B. Allsop. Sigcap: A computer program for assessing the tra c capacity of signal-controlled road junctions. Traffic Eng. Control, 17:338-341, 1976.

R. B. Allsop. Sigset: A computer program for calculating traffic capacity of signal-controlled road junctions. Traffic Eng. Control, 12:58-60, 1971.

A. S. Antipin. An extraproximal method for solving equilibrium programming problems and games. Computational Mathematics and Mathematical Physics, 45 (11):1983-1914, 2005a.

A. S. Antipin. An extraproximal method for solving equilibrium programming problems and games with coupled variables. Computational Mathematics and Mathematical Physics, 45(12):2020-2029, 2005b.

M. G. H. Bel. Game theory approach to measuring $\mathrm{t}$ he per-formance reliability of transport networks. Transportation Research, Part B. Methodological, 34 ( 6):533-545, 2000.

F. Boillot, J. M. Blosseville, J. B. Lesort, M. Papageorgiou, and N. Sellam. Optimal signal control of urban traffic networks. Proc. 6th IEEE International Conference Road Traffic Monitoring and Control, pages 75-79, 1992.

Ben-Akiva M. E. Chen O. J. Game-theoretic formulations of interaction between dynamic traffic control and dynamic traffic assignment. Transportation Research Record, 1617:179-188, 1998.

Organo del Gobierno del Distrito Federal. Programa integral de transporte y vialidad 2001-2006. In GACETA OFICIAL DEL DISTRITO FEDERAL, volume XII Epoca, pages 3-94. Administración Pública del Distrito Federal, Mexico City, 2002.

M. Godoy, A. Poznyak, and E Gomez. Generalization of the mangasarian-stone theorem for markov chain finite $n$-person games: Lpm-approach. Dynamic System and Applications, 12:489-508, 2003.

R. E. Gómez, K. Najim, and A. S. Poznyak. Saddle-point calculation for constrained finite markov chains. Inter- 
national Journal of Economic Dynamics and Control, 27:1833-1853, 2003.

P. B. Hunt, D. I. Robertson, and R. D. Bretherton. The scoot on-line traffic signal optimization technique. Traffic Eng. Control, 23:190-192, 1982.

G. Impotra. Signal control at individual junctions: Phasebased approach. Consice Encyclopedia of Traffic and Transportation Systems, pages 437-478, 2002.

G. Impotra and G. E. Cantarella. Control systems design for an individual signalised junction. Transp. Res. B, 18:147-167, 1984.

D. C. Little. The synchronization of traffic signal by mixed-integer-linear-programming. Operations Research, 14:568-594, 1966.

D. C. Little, Kelson M. D., and Gartner N. H. Maxband: A program for setting signals on arteries and triangular networks. U. S. Dept. Transport of Washington DC Transport Research Record, (795), 1981.

E. M. McDonald and N. B. Hounsell. Road traffic control: Transyt and scoot. Consice Encyclopedia of Traffic and Transportation Systems, pages 386-391, 1991.

John Forbes Nash. Equilibrium points in n-person games. Proc. Nat. Acad. U.S.A., 36:48-49, 1950.

M. Papageorgiou, C. Diakaki, V. Dinopoulou, A Kotsialos, and Wang Y. Review of road traffic control strategies. IEEE, 91(12):2041-2067, December 2003.

A. Poznyak and K. Najim. Learning through reinforcement for n-person repeated game with constraints. In Special issue on Learning Automata Theory, volume 32, pages 759-771. IEEE Trans. on Systems Man and Cybernetics, 2002.

A Poznyak and K. Najim. Self-learning Control of Finite Markov Chains. Marcel Dekker, New York, 2000.

D. I. Robertson. Transyt method for area traffic control. Traffic Eng. Control, 10:276-281, 1969.

Dennis I. Robertson and R. David Bretherton. Optimizing networks of traffic signals in real time - the sccot method. In IEEE Transactions on Vehicular Technology, volume 40, pages 11-15. IEEE Vehicular Technology Society, 1991.

J.B. Rosen. Existence and uniqueness of equilibrium points for concave n-persons games. Econometrica, 33: 520-534, 1965.

S. Sen and L. Head. Controlled optimization of phases at an intersection. Transp. Sci., 31:5-17, 1997.

K. Tanaka and K. Yokoyama. On $\varepsilon$-equilibrium point in a noncooperative $n$-person game. Journal of Mathematical Analysis and Applications, 160:413-423, 1991. 